\section{Fractional Bilinear Transform for Analog-to-Digital Conversion}

Soo-Chang Pei, Fellow, IEEE, and Hong-Jie Hsu

\begin{abstract}
In this correspondence, we propose a fractional bilinear transform used in analog-to-digital (A/D) conversion. This transfer function is derived by means of fractional delay filters. According to this transformation, first-order differentiator can be easily designed. Next, some design examples are illustrated to show the linear frequency mapping property when doing A/D conversion. Finally, the designed low-order digital differentiator is compared with the existing differentiators.
\end{abstract}

Index Terms-Digital differentiator, fractional bilinear transform, fractional delay filter, linear transformation.

\section{INTRODUCTION}

When designing a digital filter, impulse invariance, step invariance, and bilinear transform [1] are commonly used to perform analog-to-digital conversion. The bilinear transform is the most extensively adopted transformation when an analog filter frequency response is known. In dealing with a digital low-pass filter design, when an analog Butterworth filter [1] is transformed to a digital one using the bilinear transform, the transition band of the resulting filter will become narrower and sharper.

Digital differentiator is widely used in electrocardiogram processing, edge detection in image processing, and the velocity and acceleration in radar systems. Recently, there are several design methods such as rectangular-trapezoidal differentiator [4], wideband differentiator [5], designed for linear phase low-pass case [7], and finite-impulse-response (FIR) differentiator using fractional delay filter [8]. Most of these differentiators fit the ideal differentiator well in the low and middle frequency but have larger magnitude distortion in the high-frequency range.

Alternatively, fractional delay filter have been a common device in synchronization of digital receivers, incommensurate sampling rate conversion, speech processing, analog-to-digital (A/D) conversion, and sound synthesis of musical instruments. Methods for designing digital fractional delay are presented in [2], [3], and [9]-[11]. The approximation of fractional delay filter can be assorted into two categories. One is FIR filter, which has better group-delay response; the other is the infinite-impulse-response (IIR) filter, which has good magnitude approximation since it is an all-pass filter indeed. Here, we will propose new A/D transforms for digital filter design based on fractional delay filters.

In this correspondence, we first present an ideal mathematical model which has satisfactory linear property in Section II. The fractional delay filter is applied to implement the ideal model in Section III, and some examples and problems are illustrated and discussed. In Section IV, a first-order differentiator designed from the fractional bilinear transform is compared with the existing differentiators. Finally, conclusions are made.

\section{Motivation}

In analog-to-digital design, the bilinear transform is a transformation between the variables $s$ and $z$, which maps $j \Omega$ axis in the $s$-plane to the unit circle in the $z$-plane. It is a nonlinear tangent curve transformation

Manuscript received March 24, 2007; revised October 1, 2007. The associate editor coordinating the review of this manuscript and approving it for publication was Dr. Shahram Shahbzpanahi. This work was supported by National Science Council, R.O.C., under Contract 96-2221-E-089 and Contract 96-2752-E-002-006-PAE.

The authors are with the Department of Electrical Engineering, National Taiwan University, Taipei, Taiwan 10617, R.O.C. (e-mail: pei@cc.ee.ntu.edu. tw).

Digital Object Identifier 10.1109/TSP.2007.912250

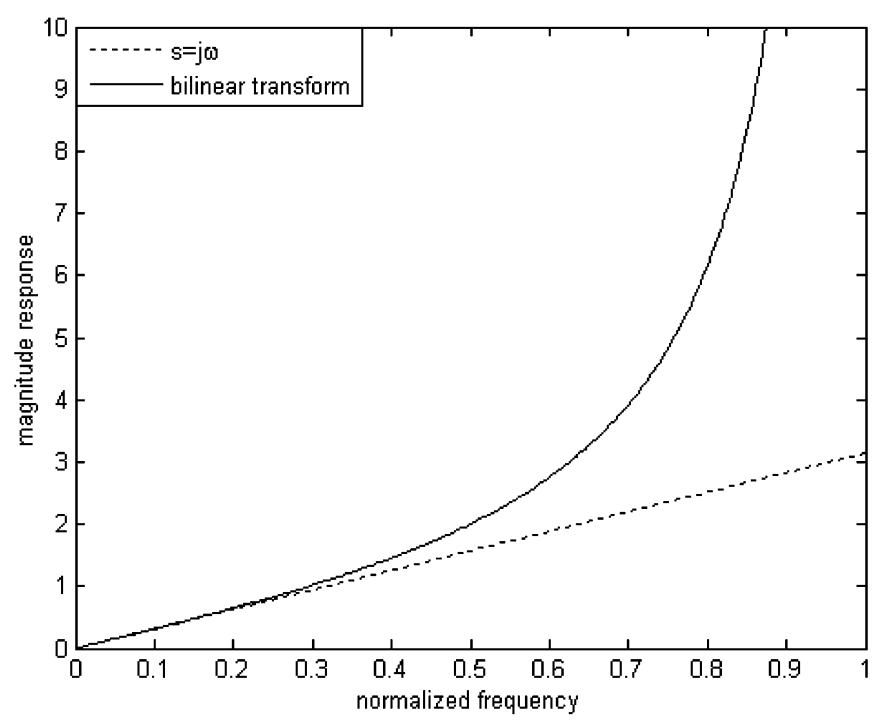

Fig. 1. Magnitude responses of $s=j \omega$ and bilinear transform $H(z)$.

(1) since the range $-\infty \leq \Omega \leq \infty$ in the continuous frequency-domain maps onto $-\pi \leq \omega \leq \pi$ in the discrete-frequency domain. Fig. 1 shows the ideal $s=j \omega$ and the magnitude response of (1) with normalized frequency $v=\omega / \pi, 0 \leq v \leq 1$ and $T=1$. In the following figures, $T$ is set to 1 if there is no other assignment. It is clear that the bilinear transformation has large distortion in high frequency range and just can be used in the situations that warping of the frequency axis is acceptable.

$$
\begin{aligned}
s & =\frac{2}{T} \frac{1-z^{-1}}{1+z^{-1}} . \\
H\left(e^{j \omega T}\right) & =\frac{2}{T} \frac{1-e^{-j \omega T}}{1+e^{-j \omega T}}=\frac{2}{T} \frac{e^{j \frac{\omega}{2} T}-e^{-j \frac{\omega}{2} T}}{e^{j \frac{\omega}{2} T}+e^{-j \frac{\omega}{2} T}}\left(\frac{e^{-j \frac{\omega}{2} T}}{e^{-j \frac{\omega}{2} T}}\right) \\
& =\frac{2}{T} j \tan \left(\frac{\omega}{2} T\right) .
\end{aligned}
$$

Fact: The following transfer function $F(z)$ [6] approaches $j \omega$ if $d$ approaches zero:

$$
F(z)=\frac{2}{d T} \frac{1-z^{-d}}{1+z^{-d}}
$$

Proof: Substituting $e^{j \omega T}$ for $z$ in (2), we get

$$
\begin{aligned}
F\left(e^{j \omega T}\right) & =\frac{2}{d T} \frac{\left(e^{j \omega \frac{d}{2} T}-e^{-j \omega \frac{d}{2} T}\right) e^{-j \omega \frac{d}{2} T}}{\left(e^{j \omega \frac{d}{2} T}+e^{-j \omega \frac{d}{2} T}\right) e^{-j \omega \frac{d}{2} T}} \\
& =\frac{2}{d T} j \tan \frac{\omega d}{2} T .
\end{aligned}
$$

Let $d$ approach zero at both sides, then (3) yields

$$
\lim _{d \rightarrow 0} F\left(e^{j \omega T}\right)=j \omega .
$$

The proof is completed.

The above fact means that the transfer function in (2) has linear magnitude response if small number $d$ approaches zero. Besides, the left-half $s$-plane is also mapped into the interior of the unit circle in the $z$-plane. It is similar to the bilinear transform with $z^{-1}$ replaced by $z^{-d}$, which will change the period of frequency from $2 \pi$ into $2 \pi / \mathrm{d}$. However, we can not implement (2) because the term $z^{-d}$ is not an integer delay. It is not possible to be realized in practice. In the following section, two design methods can approximate this fractional delay. One 
is the FIR approximation; the other is the IIR all-pass filter approximation.

\section{FRACTIONAL BILINEAR TRANSFORM}

In this section, we introduce FIR and IIR fractional delay filter to design $F(z)$ in (2). Some simulation results and problems are illustrated and discussed. The design details are described below.

\section{A. Lagrange FIR Fractional Delay Filter Case}

Lagrange interpolation method for approximating a fractional delay $z^{-(I+d)}[2],[11]$ is used as the following:

$$
z^{-(I+d)} \approx \sum_{n=0}^{N} h(n) z^{-n}
$$

where the impulse response $h(n)$ is calculated by

$$
h(n)=\prod_{m=0, m \neq n}^{N} \frac{I+d-m}{n-m} .
$$

It is clear that we have the three parameters, $I, d$, and $N$, to tune the frequency response of fractional bilinear transform where $I$ is the integer part of the delay filter, $d$ is the fractional part of the delay filter, $0<d<1$, and $N$ is the order of the FIR filter. Substituting (5) and (6) into (2), we have

$$
\begin{aligned}
F(z) & =\frac{2}{d T} \frac{1-z^{-d}}{1+z^{-d}}=\frac{2}{d T} \frac{1-z^{-d}}{1+z^{-d}}\left(\frac{z^{-I}}{z^{-I}}\right) \\
& =\frac{2}{d T} \frac{z^{-I}-z^{-(I+d)}}{z^{-I}+z^{-(I+d)}} \\
& =\frac{2}{d T} \frac{z^{-I}-\sum_{n=0}^{N}\left(\prod_{m=0, m \neq n}^{N} \frac{I+d-m}{n-m}\right) z^{-n}}{z^{-I}+\sum_{n=0}^{N}\left(\prod_{m=0, m \neq n}^{N} \frac{I+d-m}{n-m}\right) z^{-n}} .
\end{aligned}
$$

Here, we show a first-order design example when the parameters are chosen as $I=0, d=0.1$, and $N=1$ where $N$ controls the maximally flat range of the fractional group delay and $I \simeq N / 2$. Substituting into (5), (6) and (7), the resulting functions become (8) and (9), where we simplified the fractional delay to integer delay approximation. Fig. 2 shows the magnitude response of the designed fractional bilinear transform (8) compared with ordinary bilinear transform and the ideal $j \omega$ line. Note that the multiplier in (8) is 21 , which is slightly different from $20(2 / 0.1)$ since the realization has little bias from the theoretical value so we tuned the multiplier to minimize the absolute magnitude error. We can see that the line is no longer linear around $0.5 \pi$ in Fig. 2 and does not go to infinity as conventional bilinear transform.

$$
\begin{aligned}
F_{1}(z) & =\frac{21}{T} \frac{0.1-0.1 z^{-1}}{1.9+0.1 z^{-1}} . \\
z^{-0.1} & \approx 0.9+0.1 z^{-1}
\end{aligned}
$$

The next design example shows better performance. Parameters are chosen as $I=1, d=0.7$, and $N=2$, and thus it is a second-order example. Fig. 3 shows the magnitude response of the designed fractional bilinear transform (10) compared with ordinary bilinear transform and the ideal $j \omega$ line. The Lagrange approximation of $z^{-1.7}$ is given in (11). It is clear that the magnitude response fits ideal $j \omega$ well except $\omega \geq 0.9 \pi$. Similarly, the multiplier in (10) is 3.0572 instead of 2.8571

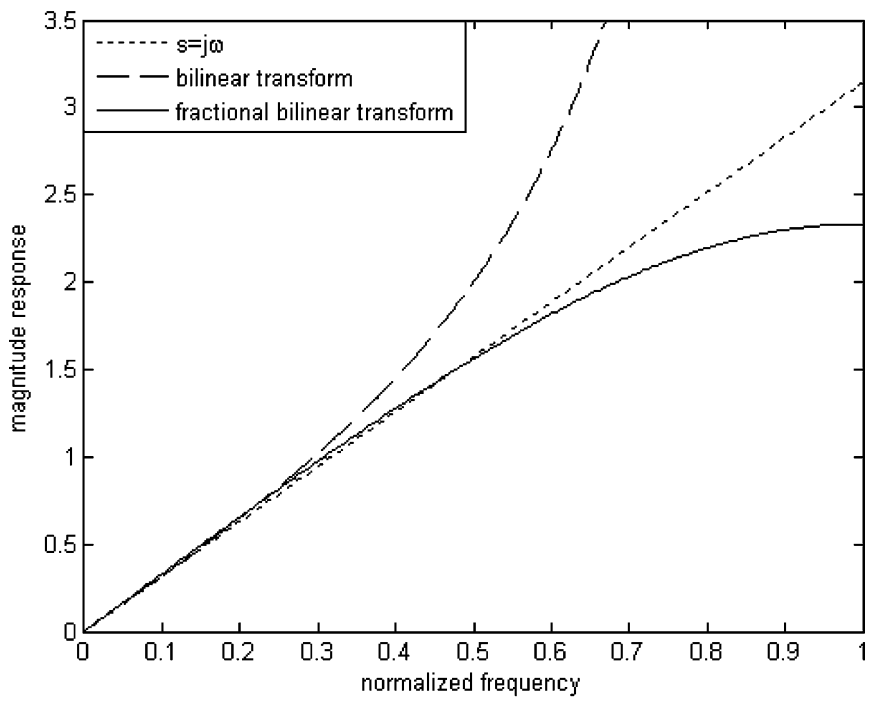

Fig. 2. Magnitude responses of $s=j \omega$, bilinear transform $H(z)$, and fractional bilinear transform $F_{1}(z)$ with parameters $I=0, d=0.1$, and $N=1$.

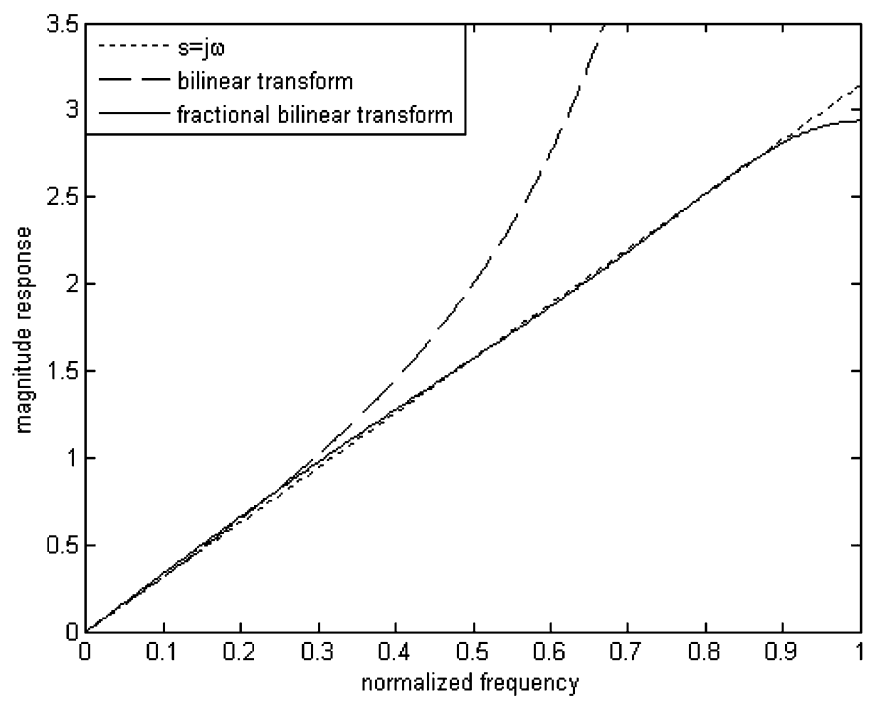

Fig. 3. Magnitude responses of $s=j \omega$, bilinear transform $H(z)$, and fractional bilinear transform $F_{2}(z)$ with parameters $I=1, d=0.7$, and $N=2$.

(2/0.7) since we tuned the multiplier to minimize the absolute magnitude error.

$$
\begin{aligned}
& F_{2}(z)=\frac{3.0572}{T} \frac{0.1050+0.4900 z^{-1}-0.5950 z^{-2}}{-0.1050+1.5100 z^{-1}+0.5950 z^{-2}} \\
& z^{-1.7} \approx-0.1050+0.5100 z^{-1}+0.5950 z^{-2}
\end{aligned}
$$

This example is a good realization of the ideal case. Nevertheless, $d=$ 0.7 is chosen instead of theoretical value $d \rightarrow 0$ because the magnitude of the Lagrange fractional delay filter is not 1 over the whole Nyquist frequency range.

Consequently, when doing analog-to-digital conversion, bilinear transform has large frequency warping effect. That is, because the magnitude response of bilinear transform goes to infinity, the analog frequency approaching $\infty$ mapped onto digital frequency $\pi$ during A/D conversion. Therefore, the high-frequency region in analog 


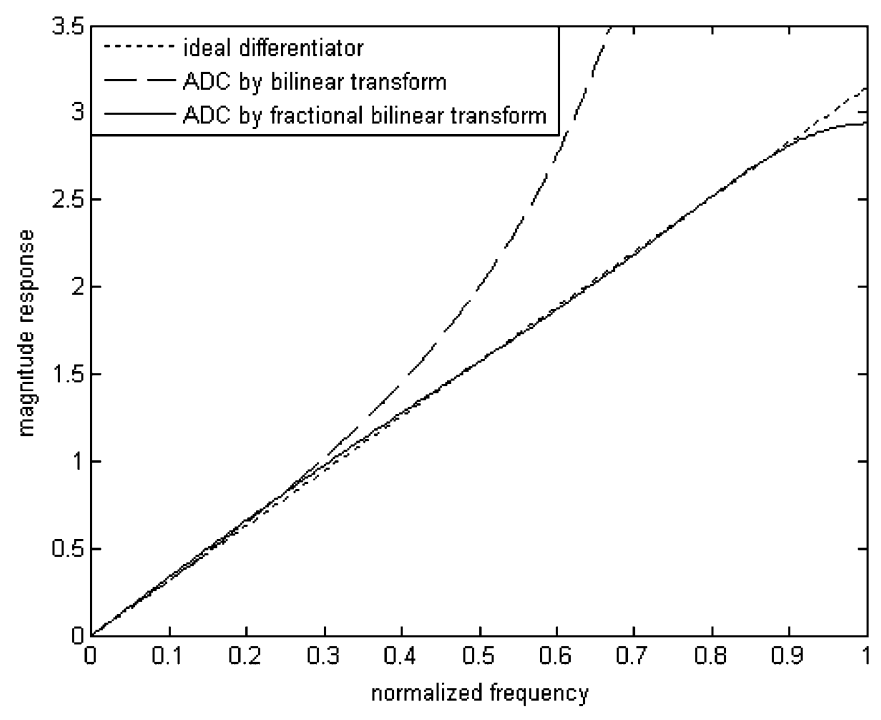

Fig. 4. Magnitude responses of ideal first-order differentiator, A/D conversion by bilinear transform, and A/D conversion by fractional bilinear transform.

domain is severely compressed, distorted, and hard to control the mapping property. However, performing A/D conversion by this proposed second-order fractional bilinear transform has much better linear mapping. It is important that $F_{1}(z)$ in (8) is stable but $F_{2}(z)$ in (10) has one unstable pole. Hence, the filter $F(z)$ in (7) may be unstable for any integer $I$ and fractional number $d$. We can use an all-pass filter to reflect the unstable pole inside the unit circle, then the final filter is stable.

Some applications of the proposed transformation are presented. The analog first-order differentiator is $s=j \omega$, and then we do bilinear transform and fractional bilinear transform, resulting (1) and (10) respectively. Equation (10) is multiplied by an all-pass filter $\left(1-14.7647 z^{-1}\right) /\left(-14.7647+z^{-1}\right)$ to ensure that all poles are inside the unit circle. The proposed first-order digital differentiator is given by

$$
\begin{aligned}
F_{\text {diff } 1-\text { frac }}(z)=\left(\frac{3.0572}{-}\right) & \\
& \quad \times \frac{0.1050+0.4900 z^{-1}-0.5950 z^{-2}}{-0.1050-0.0332 z^{-1}+0.0027 z^{-2}} .
\end{aligned}
$$

Fig. 4 shows the designed results. Similar to first-order design example, the analog second-order differentiator, $s^{2}=(j \omega)^{2}$, is transformed by the bilinear transform and $F(z)$ in (7). We obtain the same result either using two cascade first-order approach or direct second-order approach in $F(z)$ with parameters $I=1, d=0.7$, and $N=2$ (for low complexity). After stabilization process, the resulting transfer functions are depicted in (13) and (14), and the magnitude responses are shown in

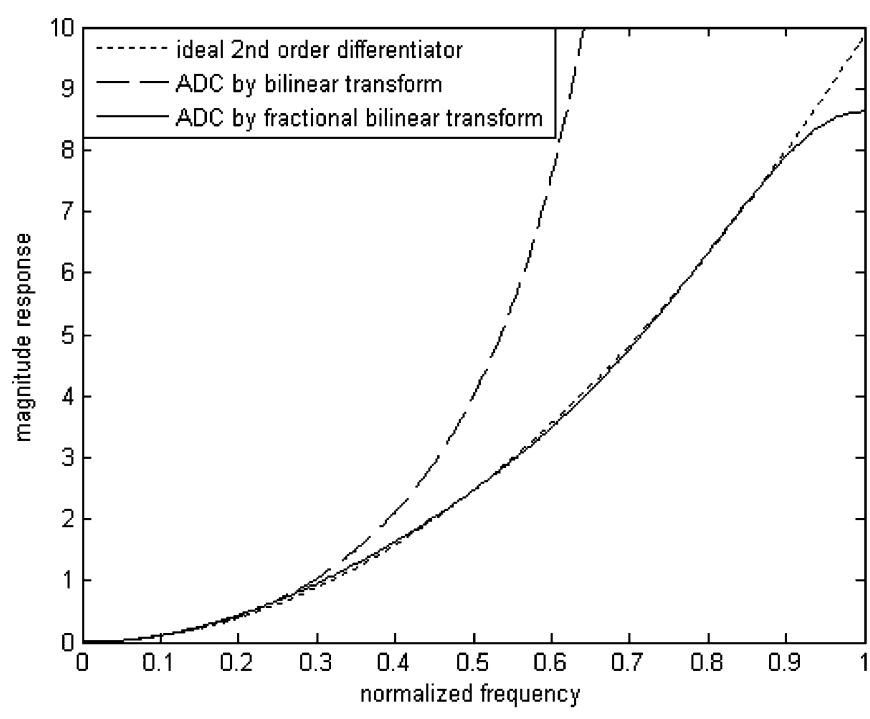

Fig. 5. Magnitude responses of ideal second-order differentiator, A/D conversion by bilinear transform, and A/D conversion by fractional bilinear transform.

Fig. 5 compared with the ideal case. [See (13) and (14), shown at the bottom of the page.]

In the first-order integrator case, the analog frequency response is $s^{-1}=(j \omega)^{-1}$, and the design results are (15) and (16) (which includes similar approach in (12) to make all poles inside the unit circle), shown in Fig. 6. Here, we choose $d=0.8$ because in low-frequency region, $d=0.7$ case has more distortion than $d=0.8$ case since small distortion in differentiator may result in large distortion in integrator in low-frequency range.

$$
\begin{aligned}
F_{\text {int-bi }}(z) & =\left(\frac{2}{T} \frac{1-z^{-1}}{1+z^{-1}}\right)^{-1}=\frac{T}{2} \frac{1+z^{-1}}{1-z^{-1}} . \\
F_{\text {int-frac }}(z) & =(-0.0444 T) \frac{-0.0800+1.3600 z^{-1}+0.7200 z^{-2}}{0.0800-0.0711 z^{-1}-0.0089 z^{-2}} .
\end{aligned}
$$

Fig. 7 is an illustrative digital filter design example of linear transform. The dashed line in Fig. 7 represents the fifth-order analog Butterworth low-pass filter. By doing bilinear transform and the proposed transform (10), respectively, we can see the shape of the solid line is more similar and close to analog LPF than the dashed-dotted line. Because bilinear transform will compress the frequency axis, the transition band is sharper, but this frequency mapping is distorted. That is why linear transform is demanded.

In summary, the proposed transform may have other useful applications when linear frequency mapping is concerned and may have better

$$
\begin{aligned}
F_{\text {diff } 2-\text { bi }}(z) & =\left(\frac{2}{T} \frac{1-z^{-1}}{1+z^{-1}}\right)^{2}=\frac{4}{T^{2}} \frac{1-2 z^{-1}+z^{-2}}{1+2 z^{-1}+z^{-2}} \\
F_{\text {diff } 2-\text { frac }}(z) & =\left(\left(\frac{3.0572}{-14.7467 T}\right) \frac{0.1050+0.4900 z^{-1}-0.5950 z^{-2}}{-0.1050-0.0332 z^{-1}+0.0027 z^{-2}}\right)^{2} \\
& =\frac{0.0430}{T^{2}} \frac{0.0110+0.1029 z^{-1}+0.1151 z^{-2}-0.5831 z^{-3}+0.3540 z^{-4}}{0.0110+0.0070 z^{-1}+0.0005 z^{-2}-0.0002 z^{-3}+0.0000 z^{-4}}
\end{aligned}
$$




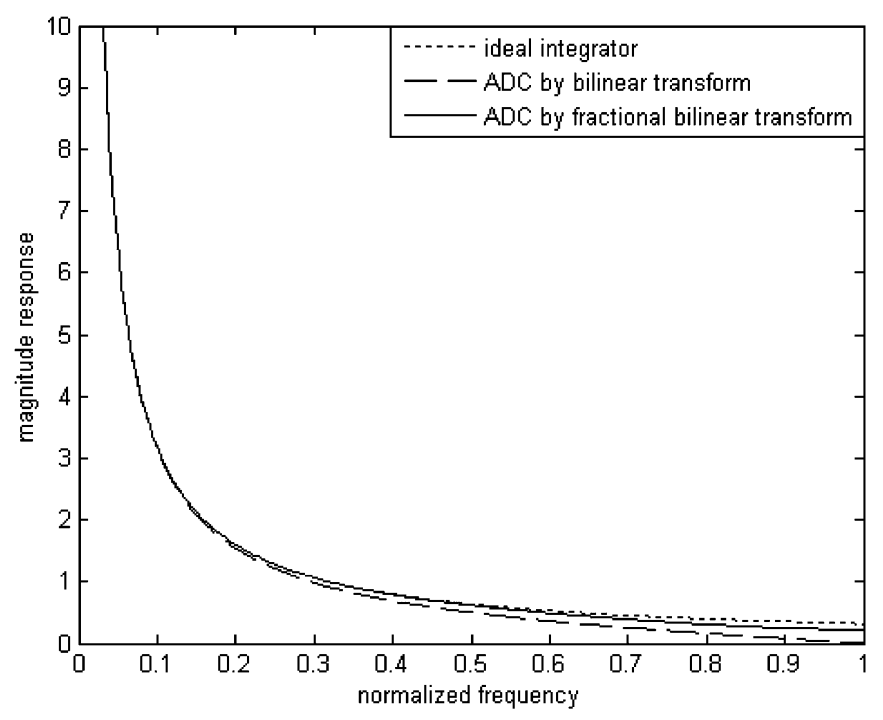

Fig. 6. Magnitude responses of ideal first-order integrator, A/D conversion by bilinear transform, and A/D conversion by fractional bilinear transform.

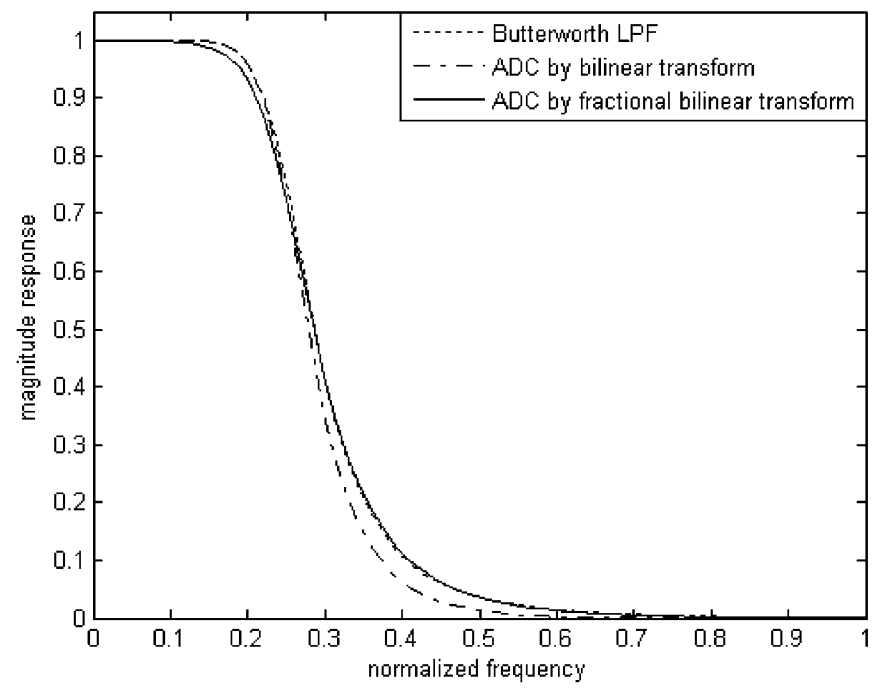

Fig. 7. Magnitude responses of Butterworth LPF, A/D conversion by bilinear transform, and A/D conversion by fractional bilinear transform.

linear property than (10) but the higher order numerator and denominator are needed.

\section{B. Thiran IIR All-Pass Fractional Delay Filter Case}

The maximally flat group delay all-pass filter for approximating a fractional delay $z^{-(I+d)}$ [2], [10] is used as (17), where the parameters $a(n)$ are calculated as in (18). Similar to FIR design methodology, (2) can be implemented as (19) where the fractional delay filter $z^{-(I+d)}$ is replaced by an IIR all-pass filter (17). Substituting (17) and (18) into (19), here we use an example to explain the design method. Parameters are chosen as $I=1, d=0.1$ and $N=15$, where $I$ is the integer part of the delay filter, $d$ is the fractional part of the delay filter, $0<d<1$, and $N$ is the order of the IIR filter. The design result is shown in Fig. 8. Here, $d$ is consistent with the ideal condition given in (4).

$$
z^{-(I+d)} \approx \frac{a(N)+a(N-1) z^{-1}+\ldots+a(0) z^{-N}}{a(0)+a(1) z^{-1}+\ldots+a(N) z^{-N}}
$$

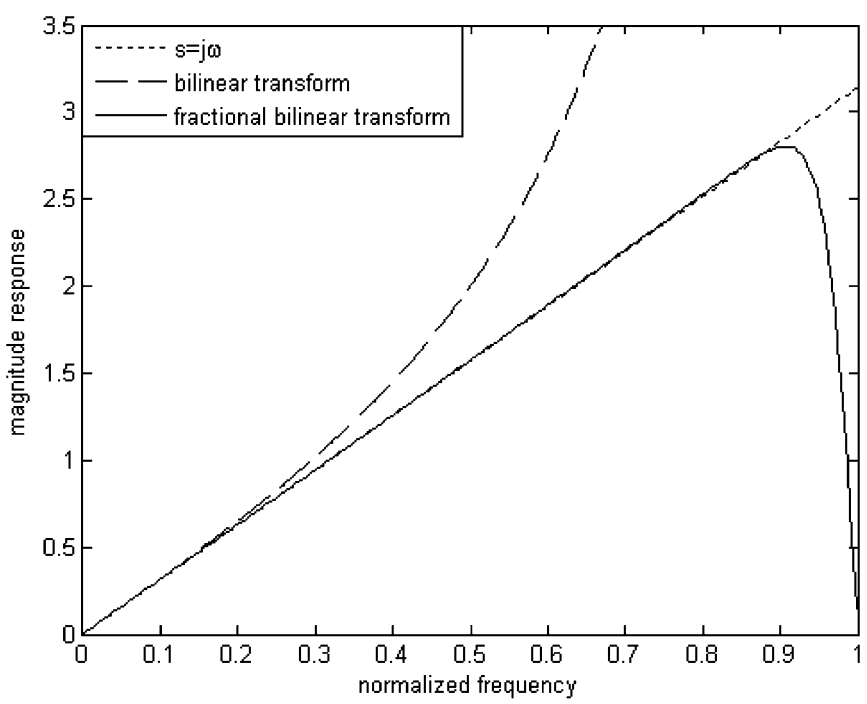

Fig. 8. Magnitude responses of $s=j \omega$, bilinear transform $H(z)$, and $F(z)$ approximated by Thiran all-pass IIR fractional delay filter with parameters $I=$ $1, d=0.1$ and $N=15$.

TABLE I

FOUR CASES OF FRACTIONAL BILINEAR TRANSFORM USING IIR FRACTIONAL DELAY FILTER AT $\omega=\pi$

\begin{tabular}{llll}
\hline If $I \in$ odd & and & $N \in$ odd, & $F\left(e^{j \pi}\right)=0$. \\
\hline If $I \in$ even & and & $N \in$ even, & $F\left(e^{j \pi}\right)=0$. \\
\hline If $I \in$ odd & and & $N \in$ even, & $F\left(e^{j \pi}\right) \rightarrow \infty$. \\
\hline If $I \in$ even & and & $N \in$ odd, & $F\left(e^{j \pi}\right) \rightarrow \infty$. \\
\hline
\end{tabular}

$$
a(n)=(-1)^{n} C_{n}^{N} \prod_{k=0}^{N} \frac{I+d-N+k}{I+d-N+n+k}
$$

where $C_{n}^{N}=N ! / n !(N-n) !$ is a binomial coefficient

$$
\begin{aligned}
F(z) & =\frac{2}{d T} \frac{1-z^{-d}}{1+z^{-d}}=\frac{2}{d T} \frac{1-z^{-d}}{1+z^{-d}}\left(\frac{z^{-I}}{z^{-I}}\right) \\
& =\frac{2}{d T} \frac{z^{-I}-z^{-(I+d)}}{z^{-I}+z^{-(I+d)}} .
\end{aligned}
$$

Consider $\omega=\pi$ in (19). If $N \in$ odd, then $z^{-(I+d)}$ in (17) and (19) yields to -1 since $e^{-j \pi}=-1$, and the sum of numerator and the sum of denominator are the same except a scalar -1 . Also, if $I \in$ odd, then $z^{-I}=-1$. Therefore, (19) reduces to

$$
F\left(e^{j \pi}\right)=\frac{2}{d}\left(\frac{-1-(-1)}{-1+(-1)}\right)=0 .
$$

We can see why Fig. 8 becomes zero at $\omega=\pi$. There are a total of four cases described in Table I.

Consequently, implementation of (2) using an IIR fractional delay filter tends toward either zero or infinity at $\omega=\pi$. The above example can be used to design a first-order digital differentiator by A/D conversion from first-order analog differentiator with frequency response $s$ and then use a similar approach in (12) to make all poles inside the unit circle by multiplying an all-pass filter. The magnitude response of the resulting low-pass differentiator is the same as the solid line in Fig. 8. However, the design order is 16 in both numerator and denominator since the parameter of the all-pass fractional delay filter is chosen as $N=15$ and $I=1$. The absolute magnitude error in the low-frequency 


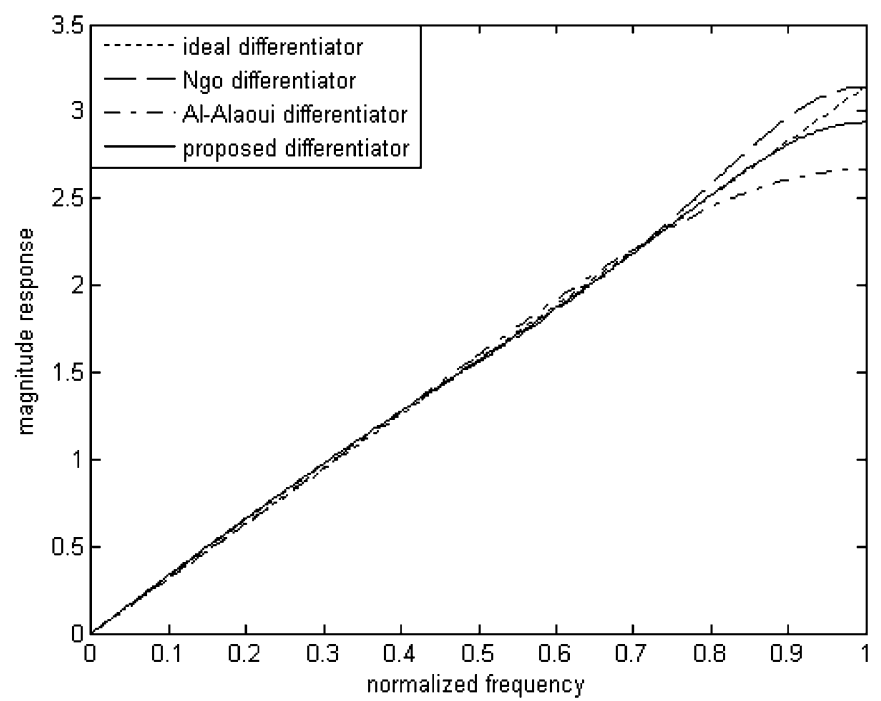

Fig. 9. Magnitude responses of ideal differentiator, Ngo's differentiator $F_{\mathrm{Ngo}}(z)$, Al-Alaoui's differentiator $F_{\mathrm{Al}-\text { Alaoui }}(z)$, and the proposed differentiator (12) transformed by fractional bilinear transform.

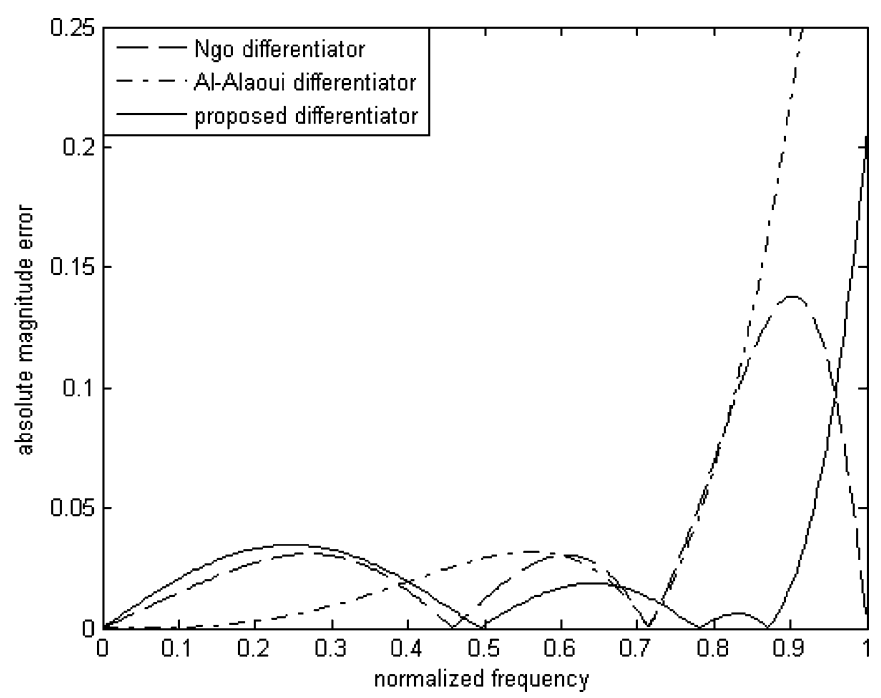

Fig. 10. Absolute magnitude errors of Ngo's differentiator $F_{\text {Ngo }}(z)$, Al-Alaoui's differentiator $F_{\mathrm{Al}-\text { Alaoui }}(z)$, and the proposed differentiator (12) transformed by fractional bilinear transform.

region is smaller than (12), but the disadvantage is higher implementation complexity. As a result, it is interesting to tune the parameters to optimize the Thiran-case fractional bilinear transform with small $N$.

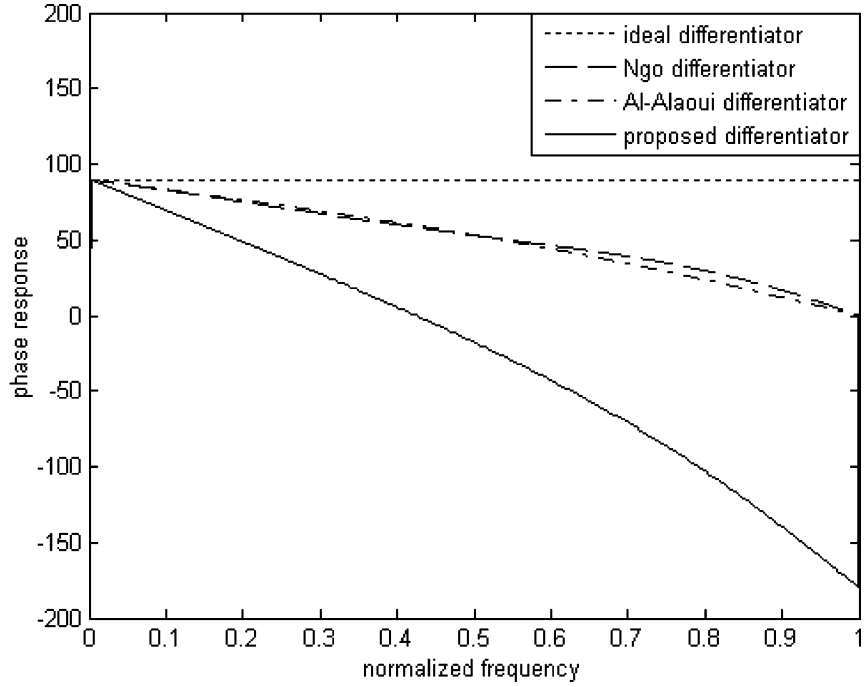

Fig. 11. Phase responses of ideal differentiator, Ngo's differentiator $F_{\mathrm{Ngo}}(z)$, Al-Alaoui's differentiator $F_{\mathrm{Al}-\mathrm{Alaoui}}(z)$, and the proposed differentiator (12) transformed by fractional bilinear transform.

\section{COMPARISON WITH SOME EXISTING DIFFERENTIATORS}

In this section, we compare the digital differentiator (12) designed from fractional bilinear transform with the rectangular-trapezoidal differentiator proposed by Al-Alaoui (21) [4] and the wideband differentiator proposed by Ngo (22) [5]. Magnitude responses and absolute magnitude errors are shown in Figs. 9 and 10, respectively. It is clear that the solid line in Fig. 10 has a much smaller error in range $0.7 \pi \leq \omega \leq 0.9 \pi$ than the others and is similar to the dashed line in range $0 \leq \omega \leq 0.5 \pi$. In addition, the absolute error function is defined in (23), which is the integral of the absolute magnitude error. Thus, substituting the proposed differentiator (12), (21), and (22) into $D\left(e^{j \omega}\right)$, yields $0.0740,0.1872$, and 0.1165 , respectively. Hence, the proposed differentiator (12) outperforms (21) and (22) in magnitude error sense. Note that the implementation complexity are second-order, first-order, and third-order, respectively. On the other hand, the comparison of phase response is described in Fig. 11. All of the three differentiators are approximately linear phase, and the ideal case is constant phase $\left(+90^{\circ}\right)$. [See $(21)-(23)$, shown at the bottom of the page.]

\section{CONCLUSION}

In this correspondence, we propose a novel approach to design fractional bilinear transform (also first-order digital differentiator) by using Lagrange fractional delay filter. Conventional bilinear transform may bring huge distortion in high frequency range and compress the analog frequency axis, resulting nonlinear effects in some A/D conversions. Our approach can help and keep the linearity properties remained while performing ADC. This fractional sample delay method is also very

$$
\begin{aligned}
F_{\mathrm{Al}-\text { Alaoui }}(z) & =\frac{8}{7 T} \frac{1-z^{-1}}{1+z^{-1 / 7}} . \\
F_{\mathrm{Ngo}}(z) & =\frac{2.7925}{2.3658 T} \frac{\left(1-z^{-1}\right)}{\left(1+1 / 2.3658 z^{-1}\right)\left(1-0.2167 e^{j 0.9427} z^{-1}\right)\left(1-0.2167 e^{-j 0.9427} z^{-1}\right)} \\
E & =\int_{0}^{\pi}|| D\left(e^{j \omega}\right)|-| j \omega|| d \omega .
\end{aligned}
$$


flexible. Different parameters may result in different filter order and frequency responses of fractional bilinear transform in diverse applications.

\section{REFERENCES}

[1] A. V. Oppenheim, R. W. Schafer, and J. R. Buck, Discrete-Time Signal Processing, 2nd ed. Englewood Cliffs, NJ: Prentice-Hall, 1999.

[2] T. I. Laakso, V. Valimaki, M. Karjalainen, and U. K. Laine, "Splitting the unit delay: Tool for fractional delay filter design," IEEE Signal Process. Mag., vol. 13, no. 1, pp. 30-60, Jan. 1996.

[3] S. C. Pei and P. H. Wang, "Closed-form design of all-pass fractional delay filters," IEEE Signal Process. Lett., vol. 11, no. 10, pp. 788-791, Oct. 2004.

[4] M. A. Al-Alaoui, "Novel digital integrator and differentiator," Electron. Lett., vol. 29, no. 4, pp. 376-378, Feb. 1993.

[5] N. Q. Ngo, "A new approach for the design of wideband digital integrator and differentiator," IEEE Trans. Circuits Syst. II, Exp. Briefs, vol. 53, no. 9, pp. 936-940, Sep. 2006.

[6] C. C. Tseng, "Improved design of digital fractional-order differentiators using fractional sample delay," IEEE Trans. Circuits Syst. I, Reg. Papers, vol. 53, no. 1, pp. 193-203, Jan. 2006.

[7] M. A. Al-Alaoui, "Linear phase low-pass IIR digital differentiators," IEEE Trans. Signal Process., vol. 55, no. 2, pp. 697-706, Feb. 2007.

[8] C. C. Tseng, "Digital differentiator design using fractional delay filter and limit computation," IEEE Trans. Circuits Syst. I, Reg. Papers, vol. 52, no. 10, pp. 2248-2259, Oct. 2005.

[9] C. W. Farrow, "A continuously variable digital delay element," in Proc. Int. Symp. Circuits Syst., May 1988, pp. 2641-2645.

[10] J.-P. Thiran, "Recursive digital filters with maximally flat group delay," IEEE Trans. Circuit Theory, vol. CT-18, no. 6, pp. 659-664, Nov. 1971.

[11] G. S. Liu and C. H. Wei, "Programmable fractional sample delay filter with Lagrange interpolation," Electron. Lett., vol. 26, no. 19, pp. 1608-1610, Sep. 13, 1990.

\section{A Square-Root Nyquist (M) Filter Design for Digital Communication Systems}

Behrouz Farhang-Boroujeny, Senior Member, IEEE

\begin{abstract}
Designing matched transmit and receive filters whose combination satisfies the Nyquist condition is a classical problem in digital communication systems. In this correspondence, we propose a novel method for designing such filters. The proposed method is based on a cost function whose minimization leads to designs that can strike a balance between the stopband attenuation, the residual intersymbol interference (ISI), robust sensitivity to timing jitter, and/or reduced peak-to-average power ratio (PAR). An iterative algorithm for optimizing the proposed cost function is suggested and its excellent performance is shown by presenting a variety of design examples. Compared to the published works, the proposed method offers the following unique advantages. By introducing a symmetry in the filter coefficients, filters with reduced computational complexity can be designed. We also introduce a design parameter that allows one to strike a balance between the PAR and other features of the desired filter.
\end{abstract}

Index Terms-Filter design, Nyquist filters, peak-to-average power ratio (PAR), timing jitter.

Manuscript received October 19, 2007; revised October 24, 2007. The associate editor coordinating the review of this manuscript and approving it for publication was Dr. Timothy N. Davidson.

The author is with the Department of Electrical and Computer Engineering, University of Utah, Salt Lake City, UT 84112 USA (e-mail: Farhang @ece.utah. edu).

Digital Object Identifier 10.1109/TSP.2007.912892

\section{INTRODUCTION}

A classical problem in data communication is to design a pair of matched transmit and receive filters whose cascade is a Nyquist pulseshape. Mathematically, this problem is phrased as follows. We wish to design a filter $H(z)$, with real-valued coefficients, such that $G(z)=$ $H(z) H\left(z^{-1}\right)$ satisfies the Nyquist criterion

$$
\sum_{k=0}^{M-1} G\left(z e^{-j 2 \pi k f}\right)=M
$$

where $M$ is an integer called the over sampling factor. It indicates the number of filter coefficients per symbol interval. Equation (1) expresses the Nyquist criterion in the frequency domain. In the time domain, the Nyquist criterion finds the form

$$
g(n)= \begin{cases}1, & n=0 \\ 0, & n=m M, \quad m \neq 0 \\ \text { arbitrary, } & n \neq m M\end{cases}
$$

where $g(n)$ is the inverse $z$-transform of $G(z)$. Also, for our further reference later, we note that $g(n)=h(n) \star h(-n)$, where $h(n)$ is the inverse $z$-transform of $H(z)$ and $\star$ denotes convolution.

A filter $G(z)$ that satisfies (1) is called Nyquist (M), [1], [2]. Moreover, when $|z|=1, G(z)=H(z) H\left(z^{-1}\right)=|H(z)|^{2}$ or, equivalently, $|H(z)|=\sqrt{G(z)}$. Hence, we refer to $H(z)$ as a root-Nyquist (M) filter.

A design $H(z)$ that satisfies the Nyquist conditions (1) and (2) exactly is generally too restrictive and thus may not lead to a satisfactory filter. There are other aspects in a real-world design that one may wish to consider and a design that strikes a good balance between these aspects is often more desirable.

The various aspects that may be considered while designing $H(z)$ are as follows.

1. The Nyquist criterion should be satisfied as closely as possible to minimize interference among successive data symbols, when channel distortion is absent/negligible.

2. The transmission bandwidth and the stopband attenuation of $H(z)$ are system parameters. These are often dictated by a frequency mask in the relevant standards and, thus, the designed $H(z)$ must fit within the mask.

3. To provide immunity against timing jitter, the magnitude of the side-lobes of the impulse response $g(n)=h(n) \star h(-n)$ should be reduced.

4. The length of $H(z)$ should be kept as small as possible to minimize the implementation cost.

5. Also, the implementation cost of $H(z)$ will be reduced if $H(z)$ is constrained to be a linear phase filter.

6. To reduce the peak-to-average power ratio (PAR) of the modulated signal, one should design a pulse-shape $h(n)$ with a reduced tail size. Such modulated signals are useful in applications where power amplifiers with limited dynamic range are available.

Clearly, these are conflicting requirements, and one must give due consideration to the underlying tradeoffs during the design. This is what makes the design of Nyquist filters a challenging task, compared with the conventional filter design. Several techniques exist in the literature for the design of digital Nyquist and/or digital matched filters whose cascade is a Nyquist filter. Some of these works have proposed design approaches that consider items $1-4$. However, there is very limited work that addresses the problem of PAR. Also, most of the current design methods are incapable of constraining $H(z)$ to linear phase. A review of these works is presented in Section II.

The goal of this correspondence is to give a novel formulation for the design of root-Nyquist (M) filters that takes into account all the above issues and allow the designer to trade among the different aspects. By 